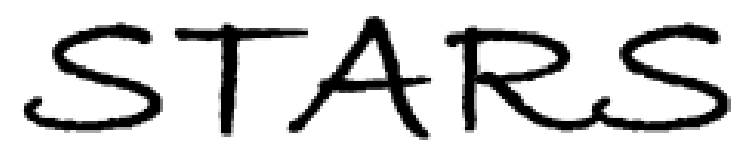

University of Central Florida

STARS

$1-1-2011$

\title{
Focus issue introduction: liquid crystal materials for photonic applications
}

Wei Lee

Shin-Tson Wu

University of Central Florida

Find similar works at: https://stars.library.ucf.edu/facultybib2010 University of Central Florida Libraries http://library.ucf.edu

This Editorial Material is brought to you for free and open access by the Faculty Bibliography at STARS. It has been accepted for inclusion in Faculty Bibliography 2010 s by an authorized administrator of STARS. For more information, please contact STARS@ucf.edu.

\section{Recommended Citation}

Lee, Wei and Wu, Shin-Tson, "Focus issue introduction: liquid crystal materials for photonic applications" (2011). Faculty Bibliography 2010s. 1529.

https://stars.library.ucf.edu/facultybib2010/1529

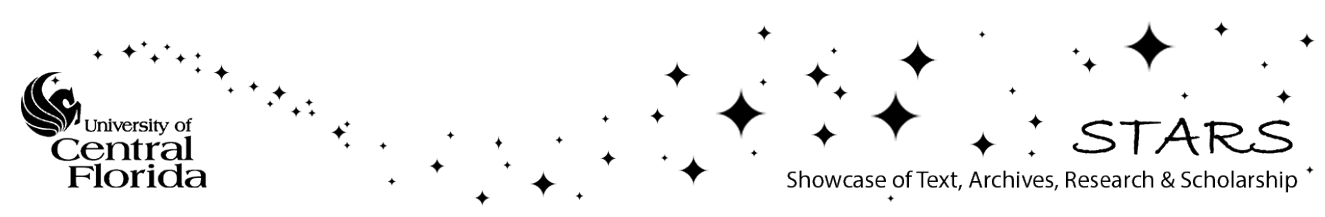




\title{
Focus issue introduction: liquid crystal materials for photonic applications
}

\author{
Wei Lee ${ }^{1, *}$ and Shin-Tson $\mathrm{Wu}^{2}$ \\ ${ }^{I}$ Department of Physics and Center for Nanotechnology, Chung Yuan Christian University, Chung-Li 32023, Taiwan \\ ${ }^{2}$ College of Optics and Photonics, University of Central Florida, Orlando 32816, Florida, USA \\ *wlee@cycu.edu.tw
}

\begin{abstract}
This special issue, "Liquid Crystal Materials for Photonic Applications," within the December 2011 publication of Optical Materials Express, highlights recent advances in liquid crystal material research. It features a collection of articles on materials and device properties of bluephase liquid crystals, dye-doped cholesteric liquid crystals, dispersions in nematic and chiral nematic liquid crystals, and of mesophase-polymer composites.

(C)2011 Optical Society of America

OCIS codes: (160.3710) Liquid crystals; (160.2100) Electro-optical materials; (160.1585) Chiral media; (160.5470) Polymers; (230.3720) Liquid-crystal devices; (230.2090) Electrooptical devices.
\end{abstract}

\section{References and links}

1. S. Elston and R. Sambles, The Optics of Theromtropic Liquid Crystals (Taylor \& Francis, 1998).

2. H.-S. Kitzerow and C. Bahr, Chirality in Liquid Crystals (Springer, 2001).

3. T. J. Sluckin, D. A. Dunmur, and H. Stegemeyer, Crystals That Flow (Taylor \& Francis, 2004).

4. F. Reinitzer, "Beiträge zur Kenntniss des Cholestherins," Monatsh. Chem. 9(1), 421-441 (1888).

5. H. Kikuchi, M. Yokota, Y. Hisakado, H. Yang, and T. Kajiyama, "Polymer-stabilized liquid crystal blue phases," Nat. Mater. 1(1), 64-68 (2002).

6. J. Yan, L. Rao, M. Jiao, Y. Li, H. C. Cheng, and S. T. Wu, "Polymer-stabilized optically isotropic liquid crystals for next-generation display and photonics applications," J. Mater. Chem. 21(22), 7870-7877 (2011).

7. J. Yan and S.-T. Wu, "Polymer-stabilized blue phase liquid crystals: a tutorial [Invited]," Opt. Mater. Express 1(8), 1527-1535 (2011), http://www.opticsinfobase.org/ome/abstract.cfm?URI=ome-1-8-1527.

8. G. Zhu, X.-W. Lin, W. Hu, Z.-G. Zheng, H.-F. Wang, H.-Q. Cui, D. Shen, and Y.-Q. Lu, "Liquid crystal blue phase induced by bent-shaped molecules with allylic end groups," Opt. Mater. Express 1(8), 1478-1483 (2011), http://www.opticsinfobase.org/ome/abstract.cfm?URI=ome-1-8-1478.

9. T. N. Oo, T. Mizunuma, Y. Nagano, H. Ma, Y. Ogawa, Y. Haseba, H. Higuchi, Y. Okumura, and H. Kikuchi, "Effects of monomer/liquid crystal compositions on electro-optical properties of polymer-stabilized blue phase liquid crystal," Opt. Mater. Express 1(8), 1502-1510 (2011), http://www.opticsinfobase.org/ome/abstract.cfm?URI=ome-1-8-1502.

10. T. Mizunuma, T. N. Oo, Y. Nagano, H. Ma, Y. Haseba, H. Higuchi, Y. Okumura, and H. Kikuchi, "Electrooptical properties of polymer-stabilized blue phase with different monomer combination and concentration," Opt. Mater. Express 1(8), 1561-1568 (2011), http://www.opticsinfobase.org/ome/abstract.cfm?URI=ome-1-8-1561.

11. S. Yabu, H. Yoshida, G. Lim, K. Kaneko, Y. Okumura, N. Uehara, H. Kikuchi, and M. Ozaki, "Dual frequency operation of a blue phase liquid crystal," Opt. Mater. Express 1(8), 1577-1584 (2011), http://www.opticsinfobase.org/ome/abstract.cfm?URI=ome-1-8-1577.

12. I. P. Ilchishin, L. N. Lisetski, and T. V. Mykytiuk, "Reversible phototuning of lasing frequency in dye doped cholesteric liquid crystal and ways to improve it [Invited]," Opt. Mater. Express 1(8), 1484-1493 (2011), http://www.opticsinfobase.org/ome/abstract.cfm?URI=ome-1-8-1484.

13. C.-T. Wang and T.-H. Lin, "Bistable reflective polarizer-free optical switch based on dye-doped cholesteric liquid crystal [Invited]," Opt. Mater. Express 1(8), 1457-1462 (2011), http://www.opticsinfobase.org/ome/abstract.cfm?URI=ome-1-8-1457.

14. C. P. Jisha, K.-C. Hsu, Y.Y. Lin, J.-H. Lin, K.-P. Chuang, C.-Y. Tai, and R.-K. Lee, "Phase separation and pattern instability of laser-induced polymerization in liquid-crystal-monomer mixtures," Opt. Mater. Express 1(8), 1494-1501 (2011), http://www.opticsinfobase.org/ome/abstract.cfm?URI=ome-1-8-1494.

15. E. Ouskova, J. Vapaavuori, and M. Kaivola, "Self-orienting liquid crystal doped with polymer-azo-dye complex," Opt. Mater. Express 1(8), 1463-1470 (2011), http://www.opticsinfobase.org/ome/abstract.cfm?URI=ome-1-8-1463. 
16. A. T. Juhl, D.-K. Yang, V. P. Tondiglia, L. V. Natarajan, T. J. White, and T. J. Bunning, "Ordering of glass rods in nematic and cholesteric liquid crystals," Opt. Mater. Express 1(8), 1536-1547 (2011), http://www.opticsinfobase.org/ome/abstract.cfm?URI=ome-1-8-1536.

17. D.-P. Cai, H.-Y. Pan, J.-F. Tsai, H.-K. Chiu, S.-C. Nian, S. H. Chang, C.-C. Chen, and C.-C. Lee, "Liquid crystal infiltrated waveguide with distributed Bragg reflectors," Opt. Mater. Express 1(8), 1471-1477 (2011), http://www.opticsinfobase.org/ome/abstract.cfm?URI=ome-1-8-1471.

Thermotropic nematic liquid crystals (LCs) are the most significant mesophase among all known liquid crystalline phases [1]. Upon heating, materials possessing mesogenic phases do not transform directly to an isotropic liquid but, instead, form thermodynamically stable phase(s) whose optical and dielectric properties are intermediate between those of a crystal and a liquid. Additional phases appear if the constituent molecules are chiral or doped with some chiral dopants, as exemplified by the blue phases (BPs) [2]. Chiral nematics are the chiral versions of nematics, sometimes referred to as the cholesteric phase since this phase was first observed in esters of cholesterol [3]. Upon cooling, cholesteric LCs (CLCs) may form up to three BPs, with BPI being the mostly encountered mesophase.

Historically from 1888 [4] through the beginning of the 21st century, studies of BPs had been restricted to pure science owing to their narrow temperature range (up to the order of magnitude of $1 \mathrm{~K}$ ) as a common characteristic in all BPs. Since the first polymer-stabilized BPs exhibiting a wider temperature range were demonstrated [5], rapid progress has been made in the development and exploration of this class of chiral LC substances. The evolutions of materials syntheses, composition, and cell fabrication as well as device designs are primarily aimed for their potential applications in photonics, giving rise to their emergence as a leading candidate for next-generation liquid crystal displays and high speed photonics based on the electro-optical Kerr effect [6]. In view of current information display technologies, their revolutionary features are, indeed, extremely alluring, including the alignment-layer-free fabrication process and short response time (in the submillisecond scale or smaller) to enable the field-sequential operation without using color filters.

There is no doubt that the publication of a special issue focusing on this hot topic, i.e., research of BPs, is timely. Five articles on BP LCs are included in this feature issue on "Liquid Crystal Materials for Photonic Applications," in which two of them pertain to new materials concepts and three are related to typical polymer-stabilized BP composites. By omitting the complicated theories in their tutorial, Yan and $\mathrm{Wu}$ [7] provide a concise, topical summary of the fundamentals, materials preparations, and display applications of polymerstabilized BPs, which is very accessible to the reader and most suitable to serve as a practical guide for those who are interested in entering this particular field of developing new LC technology. In their paper, Zhu and associates [8] explore a new approach to stabilizing a BP by doping a bent-shaped molecule with allylic end groups into a CLC mixture. The temperature range of the $\mathrm{BP}$ is reported to go over $10 \mathrm{~K}$ from $\sim 6 \mathrm{~K}$ after UV curing of the photoreactive monomer. Oo et al. [9] investigate the effect of monomer/LC composition on the electro-optical properties of BP LCs and confirm that the higher monomer content results in higher operation voltage, smaller hysteresis and residual birefringence, and shorter response time. Furthermore, the same Japanese team, Mizunuma and colleagues [10], presents a comparative study to establish the link between the electro-optical properties and the polymer type (acrylate vs. methacrylate) and concentration in a polymer-stabilized BP LC system. An interesting development of pristine BPs is reported by Yabu and coauthors [11], who demonstrate dual-frequency switching in a BP LC that is advantageous over conventional BPs in polarization-independent phase-modulation devices since a larger lattice deformation can be obtained.

In addition to the research area of BP LCs, studies of CLC materials for laser devices and flexible displays have caught a lot of attention in the past decade. The invited paper by Ilchishin, Lisetski, and Mykytiuk [12] describes a novel tunable distributed feedback laser based on a CLC composed of a mixture of cholesterol esters and azoxy nematics doped with a pyrromethene dye, leading to a dramatically high luminescence yield to ensure efficient lasing 
at the selective reflection band edge. Moreover, Wang and Lin [13] report on a short-pitched CLC doped with a dichroic dye in a homeotropic cell so to create a bistable electro-optical switch between the dark planar and bright uniformly-lying-helix states. Note that these two papers are both based on dye-doped CLC systems.

Two papers focusing on nematic-polymer hybrids are also compiled in this special issue. While Jisha et al. [14] provide a laser lithography treatment by employing an ultra-short femtosecond laser pulse to manipulate phase separation morphologies as well as pattern formation in polymer-dispersed LCs, Ouskova, Vapaavuori, and Kaivola [15] develop a dyedoped LC-polymer composite that can be used in optoelectronic applications as a simple and quite efficient photosensitive smart material entailing no additional aligning treatment.

With many possible colloidal systems of nano- and micro-scale solids in anisotropic fluids in the mesophase nowadays, numerous intriguing phenomena are awaiting theoretical and experimental exploration. In investigating the behavior of micron-sized glass rods dispersed in nematic, twisted-nematic, and CLCs with various pitch lengths, Juhl and colleagues [16] clearly present both experimental and simulation results that may lead to applications as important as self-assembled metamaterials. The paper by Cai and coauthors [17] is very much device-oriented. They demonstrate an interesting LC-infiltrated waveguide with multi-layer distributed Bragg reflectors surrounding the LC core and suggest its further applications in the scanning projection display and tunable dye-doped LC laser. 